\title{
VITAMIN A MODIFIES THE EXPRESSION OF ANTIOXIDANT ENZYMES IN THE DEVELOPING LUNG
}

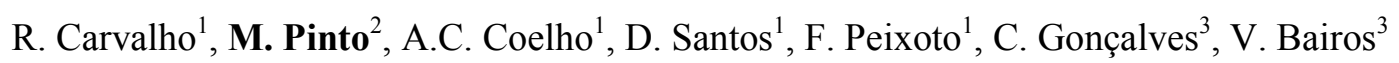 \\ ${ }^{1}$ Universidade de Trás-os-Montes e Alto Douro, ${ }^{2}$ Universidadade de Trás-os-Montes e Alto Douro, Vila \\ Real, ${ }^{3}$ Histologia e Embriologia, Facudade de Medicina - Universidade de Coimbra, Coimbra, Portugal
}

Retinol has proven to be an essential molecule during lung fetal development, but its effects on the pulmonary antioxidant system are still unknown. At birth, there is a major increase of reactive oxygen species due to the exposure to oxygen, which may lead to cellular damage. This increase is particularly important in the premature, due to the antioxidant system immaturity. To investigate the retinol influence on lung's antioxidant enzymes we conducted an in vivo study. Pregnant mice were subjected to subcutaneous administration of vitamin $\mathrm{A}$ on the $12^{\text {th }}$ gestational day. Lungs from control and treated animals were collected daily from the $15^{\text {th }}$ gestational day till the $3^{\text {th }}$ day of life. Catalase was measured by the polarographic method and glutathione peroxidase and superoxide dismutase by spectrometry. Ours results show a positive effect of vitamin A administration on both catalase and glutathione peroxidase enzymes. A statistical significance of catalase expression was reached at $16^{\text {th }}$ gestational day in vitamin A-exposed lungs. An identical difference in glutathione peroxidase expression was registered at the $15^{\text {th }}$ and $16^{\text {th }}$ gestational days. We have previously demonstrated that catalase and glutathione peroxidase levels are higher in the lungs of males in the same time-period, a feature that was not altered by vitamin A administration. Previous studies from our group also showed that maternal administration of vitamin A results in an enhancement of lung organogenesis, which can be achieved, in part, by stimulating the lung's antioxidant system, as occurs with the surfactant system, without eliminating free radicals physiological functions. 